Design and implementation of Dilation X-ray Imager for NIF "DIXI"

M. J. Ayers, S. R. Nagel, B. Felker, P. M. Bell, D. K. Bradley, K. Piston, J. Parker, Z. Lamb, J. D. Kilkenny, T. J. Hilsabeck, T. Chung, J. D. Hares, A. K. L. Dymoke-Bradshaw

September 24, 2013

SPIE Conference San Diego, CA, United States August 25, 2013 through August 29, 2013 
This document was prepared as an account of work sponsored by an agency of the United States government. Neither the United States government nor Lawrence Livermore National Security, LLC, nor any of their employees makes any warranty, expressed or implied, or assumes any legal liability or responsibility for the accuracy, completeness, or usefulness of any information, apparatus, product, or process disclosed, or represents that its use would not infringe privately owned rights. Reference herein to any specific commercial product, process, or service by trade name, trademark, manufacturer, or otherwise does not necessarily constitute or imply its endorsement, recommendation, or favoring by the United States government or Lawrence Livermore National Security, LLC. The views and opinions of authors expressed herein do not necessarily state or reflect those of the United States government or Lawrence Livermore National Security, LLC, and shall not be used for advertising or product endorsement purposes. 


\title{
Design and implementation of Dilation X-ray Imager for NIF “DIXI”
}

\author{
M.J. Ayers, S.R. Nagel, B. Felker, P.M. Bell, D.K. Bradley, K. Piston, J. Parker, Z. Lamb \\ Lawrence Livermore National Laboratory \\ J.D. Kilkenny, T.J. Hilsabeck, T. Chung \\ General Atomics \\ J.D. Hares, A.K.L. Dymoke- Bradshaw \\ Kentech Instruments Ltd.
}

\begin{abstract}
Gated X-Ray imagers have been used on many ICF experiments around the world for time resolved imaging of the target implosions. DIXI (Dilation X-ray Imager) is a new fixed base diagnostic that has been developed for use in the National Ignition Facility. The DIXI diagnostic utilizes pulse-dilation technology $[1,2,3,4]$ and uses a high magnification pinhole imaging system to project images onto the instrument. DIXI is located outside the NIF target chamber approximately $6.5 \mathrm{~m}$ from target chamber center (TCC). The pinholes are located $10 \mathrm{~cm}$ from TCC and are aligned to the DIXI optical axis using a diagnostic instrument manipulator (DIM) on an adjacent port. By use of an extensive lead and poly shielded drawer enclosure DIXI is capable of collecting data at DT neutron yields up to Yn $10^{16}$ on CCD readout and up to $\mathrm{Yn} \sim 10^{17}$ on film. Compared to existing pinhole $\mathrm{x}$-ray framing cameras DIXI also provides a significant improvement in temporal resolution, $<10 \mathrm{ps}$, and the ability to capture a higher density of images due to the fact the pinhole array does not require collimators. The successful deployment of DIXI on the NIF required careful attention to the following subsystems, pinhole imaging, debris shielding, filtering and image plate (FIP), EMI protection, large format CsI photocathode design, detector head, detector head electronics, control electronics, CCD, film recording and neutron shielding. Here we discuss the initial design, improvements implemented after rigorous testing, infrastructure and commissioning of DIXI on the NIF.
\end{abstract}

Keywords: x-ray imager, time dilation, gated, magnetic field, de-warp

\section{Introduction}

Time gated x-ray imaging [5] is essential for experiments at the National Ignition Facility (NIF) [7]. Time gated imaging provides temporally resolved images that are used for measuring shape, size and burn history of an imploding cryogenic deuterium-tritium (DT) capsule as it is compressed during Inertial Confinement Fusion (ICF) experiments.

To further study the symmetry and fuel size during implosions new time resolved x-ray imaging systems are required. The most common of these are pinhole array framing cameras that consist of a pinhole array that produces a large number of x-ray images onto a micro channel plate (MCP). The temporal gating of the MCP allows the x-ray images to be captured at different times enabling the reconstruction into a movie of the target shape size and burn history.

A similarly configured fixed based x-ray detector on the NIF, (ARIANE) Active Readout In A Neutron Environment is capable of capturing data at DT neutron yields up to $\mathrm{Yn} \sim 10^{14}$ at a standoff distance of $6.3 \mathrm{~m}$ from TCC. At the same standoff distance and switching from CCD to film readout a slight improvement is realized with the ability to capture data at yields up to $\mathrm{Yn} \sim 10^{15}$ [8]. ARIANE, similar to its predecessors GXD and HGXI are limited to 70-100ps temporal resolution. The deployment of DIXI offers significant improvement in these areas with a $7 \mathrm{x}$ reduction in temporal resolution and increased rad hardness that enables data collection on CCD at $\mathrm{Yn} \sim 10^{16}$. A summary comparing DIXI to ARIANE is shown in table 1. 


\begin{tabular}{|c|c|c|c|c|c|c|}
\hline \multicolumn{7}{|c|}{ DIXI and ARIANE Comparison On NIF } \\
\hline & $\begin{array}{l}\text { Temporal } \\
\text { Resolution } \\
\text { or Gate } \\
\text { Width } \\
\text { (ps) }\end{array}$ & Counts/Pixel & $\begin{array}{l}\text { MCP } \\
\text { Bias } \\
\text { (V) }\end{array}$ & $\begin{array}{l}\text { Events/Resolution } \\
\text { Element }\end{array}$ & Magnification & $\begin{array}{l}\text { Allowable } \\
\text { Neutron } \\
\text { Yield } \\
\text { with CCD } \\
\text { Readout }\end{array}$ \\
\hline DIXI & 10 & 26431 & 100 & 347 & 25 & $\mathrm{Yn} \sim 10^{14}$ \\
\hline ARIANE & 100 & 11000 & 400 & 495 & 65 & $\mathrm{Yn} \sim 10^{16}$ \\
\hline
\end{tabular}

Table 1. Comparison of DIXI and ARIANE on the NIF target chamber

\section{System Overview}

DIXI is located on the equator of the NIF target chamber on port $90 / 100$, see figure 1 . The DIXI diagnostic is a fixed base instrument specifically designed for high yield operations. There are several shielding improvements that allow operation at high yield, positioning of the detector at $20^{\circ}$ off the port axis which places it behind 18” of target chamber Gunite, $30 \mathrm{~cm}$ of polyethylene direct shine shielding, and a shielded enclosure surrounding the detector with $5 \mathrm{~cm}$ of lead plus $20 \mathrm{~cm}$ of additional polyethylene. To further increase the rad hardness of DIXI the pulsers and electronics are all mounted outside the target bay in the mezzanine behind the concrete walls.

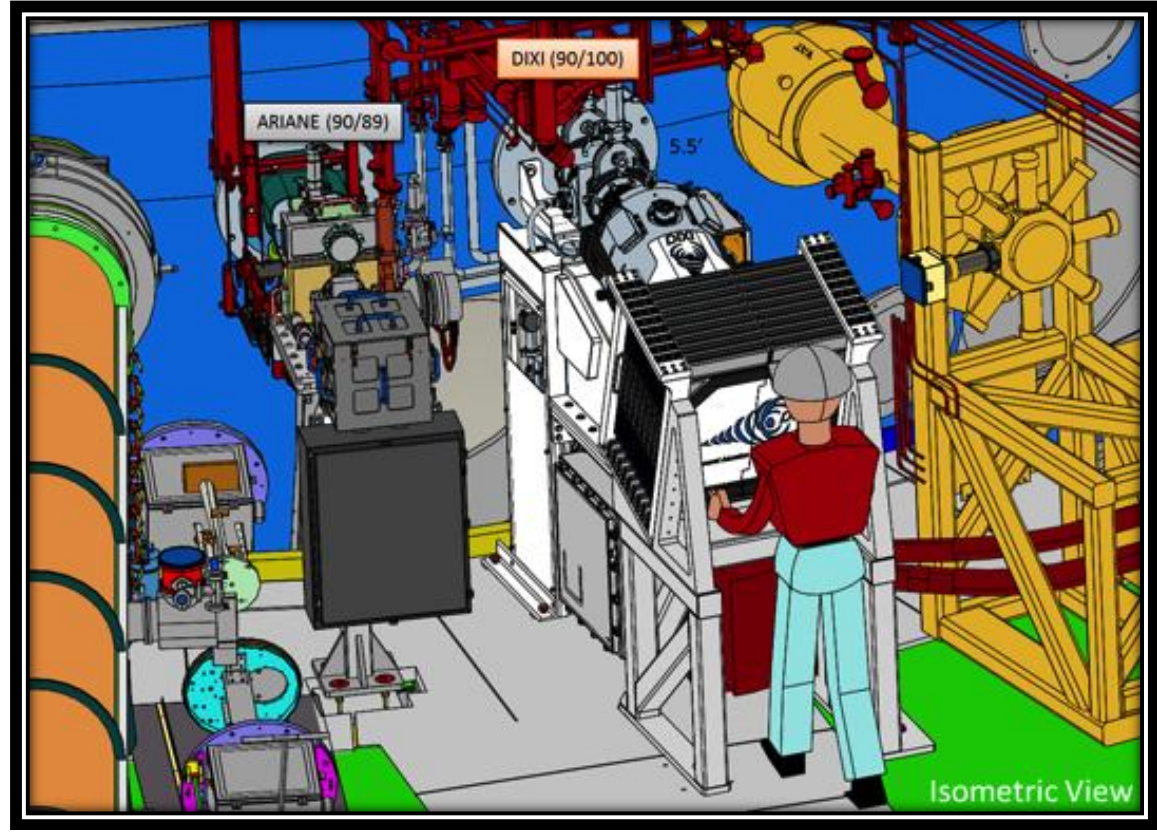

Figure 1. DIXI mounted to port 90/100 on the NIF target chamber with ARIANE shown on the left. 
The DIXI instrument is secured in an aluminum frame harness and mounted to a pedestal on an adjustable kinematic plate. The adjustment of the pedestal allows for precision alignment of the instrument in the facility. The pedestal sits on an aluminum platform suspended between 2 steel frames that are seismically anchored to the facility diving boards. The support stands are broken into 3 subassemblies: front utilities stand, bridge support with pedestal, and rear shielding stand.

The front utilities stand houses all of the DIXI vacuum utilities and is covered with aesthetic covers that use captive thumb screws for quick removal. The front utilities stand is also fitted with 2 junction boxes, one that contains the DIXI vacuum controls and a second junction box that handles the terminations of the high current magnet pulse cables. On the side nearest the operators the front utilities stand is fitted with a utilities distribution panel (UDP) that controls tempered water (TW) cooling to the systems turbo pump and CCD camera.

The bridge support spans the distance between the front utilities stand and rear shielding stand due to pre-existing utilities below.

The shielding support stand is designed to handle over 1 ton of shielding and is configured using individual drawers that allow for quick and easy access to the CCD camera and or film. Additionally mounted to the shield stand is the compressed gas UDP to lock out tag out (LOTO) valves for safe operations and a tritium sample UDP used to measure tritium levels inside the vessel prior to venting for access. A system schematic is shown in figure 2. A design summary table with component distances from TCC to detector hardware is listed in table 2.

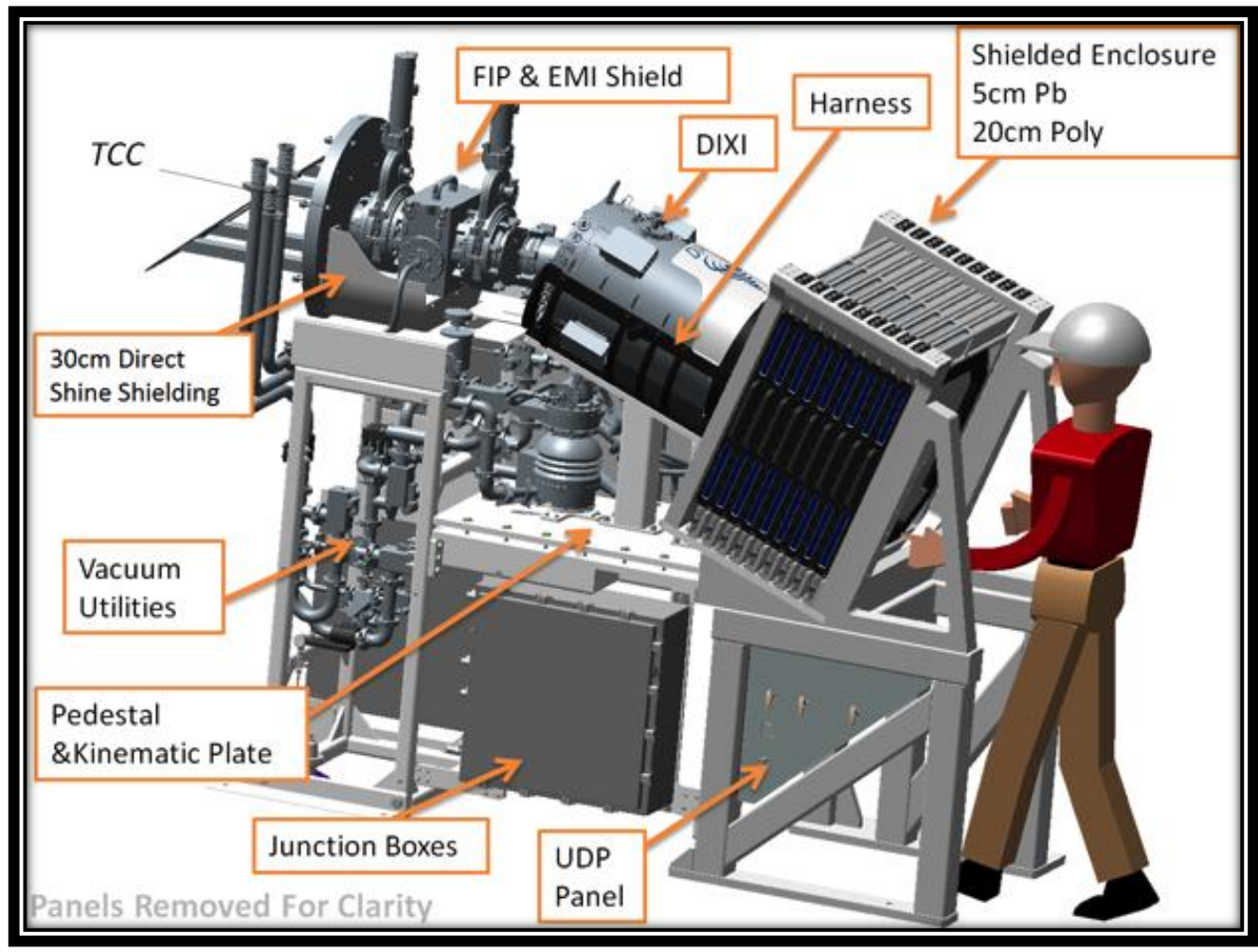

Figure 2. DIXI schematic with callouts on primary system components 
Table 2. DIXI Design Summary Table

\begin{tabular}{|l|l|l|l|l|l|}
\hline \multicolumn{6}{|l|}{ DIXI Subsystems and relative position from TCC } \\
\hline $\begin{array}{l}\text { TCC } \\
\phi\end{array}$ & $\begin{array}{l}\text { Pinhole } \\
\text { Location }\end{array}$ & FIP & EMI Protection & $\begin{array}{l}\text { Photo Cathode } \\
(\mathrm{PC})\end{array}$ & $\begin{array}{l}\text { Recording } \\
\text { backend }\end{array}$ \\
\hline $0 \mathrm{~cm}$ & $10 \mathrm{~cm}$ & $5.96 \mathrm{~m}$ & $6.02 \mathrm{~m}$ & $6.51 \mathrm{~m}$ & $7.1 \mathrm{~m}$ \\
\hline
\end{tabular}

\begin{tabular}{|c|c|c|c|c|c|c|}
\hline \multicolumn{7}{|c|}{ DIXI Detector Details } \\
\hline Pinhole & IP & EMI & $\mathrm{PC}$ & MCP & $C C D$ & FILM \\
\hline $\begin{array}{l}10 \text { um } \varnothing \\
\text { (75um } \\
\text { thick Ta) }\end{array}$ & $\begin{array}{l}\text { FUGI } \\
\text { BAS-IP } \\
\text { MS } \\
2040\end{array}$ & $\begin{array}{lr}0.5 \text { um } & \text { Al } \\
\text { vapor } & \\
\text { deposited on } \\
\text { 25um } \\
\text { Kapton }\end{array}$ & $\begin{array}{l}400 \mathrm{~nm} \text { CsI on } 200 \mathrm{~nm} \\
\text { Al on 50um Kapton. } \\
\text { MACOR support ring }\end{array}$ & $\begin{array}{l}8^{\circ} \text { Bias, } \\
\text { Gold } \\
\text { coated, } \\
\text { single } \\
\text { strip }\end{array}$ & $\begin{array}{l}\text { P43 phosphor, } \\
\text { Spectral } \\
\text { Instruments } \\
\text { SI-1000 }\end{array}$ & $\begin{array}{l}\text { P11 } \\
\text { phosphor } \\
\text { TMAX3200 }\end{array}$ \\
\hline
\end{tabular}

\section{Imaging Configuration}

A plan view of the DIXI imaging system is shown below in figure 3. The DIXI pinholes are located $10 \mathrm{~cm}$ from TCC. The DIXI pinholes are positioned and supported off a Diagnostic Instrument Manipulator (DIM). The pinholes are mounted to a custom cone that carries pinholes for 3 independent instruments, the primary DIM based instrument, ARIANE and DIXI. It is important to note that the ARIANE and DIXI pinhole mounts are static and cannot be actively aligned in the target chamber. The positioning of the ARIANE and DIXI pinholes vary from shot to shot as small adjustments are made when optimizing alignment on the primary line of sight. To compensate for these small changes in alignment additional pinholes are added to overfill the detector plane and compensate for miss-pointing. By using empirical alignment data recorded from ARIANE we were able to accurately and efficiently determine the amount of pinhole over fill required for DIXI, and were able to optimize the ARIANE pinhole array size from $12.7 \mathrm{~mm}$ diameter down to $6.35 \mathrm{~mm}$. The DIXI pinhole array requires $1.5 \mathrm{~mm}$ of additional pinholes radially to compensate for miss-pointing.

The DIXI instrument is mounted outside the target chamber with the photocathode located 6.5 $\mathrm{m}$ from TCC making DIXI a very high magnification instrument, 64x. Mounted directly to the target chamber gate valve is a filter image plate holder or (FIP). Inside the FIP there is a filter cartridge used to hold transmission filters, image plate and an EMI shield. The filters and image plates sit in front of the EMI shield and are used as a telltale for potential debris damage to the EMI shield. The EMI shield is critical to prevent unwanted background on the photo cathode that could distort the quality of the image. The photocathode and detector are separated by a $50 \mathrm{~cm}$ drift tube. The detector is located at $7.1 \mathrm{~m}$ from TCC and can be accessed by sequentially opening a series of lead and poly lined drawers. 


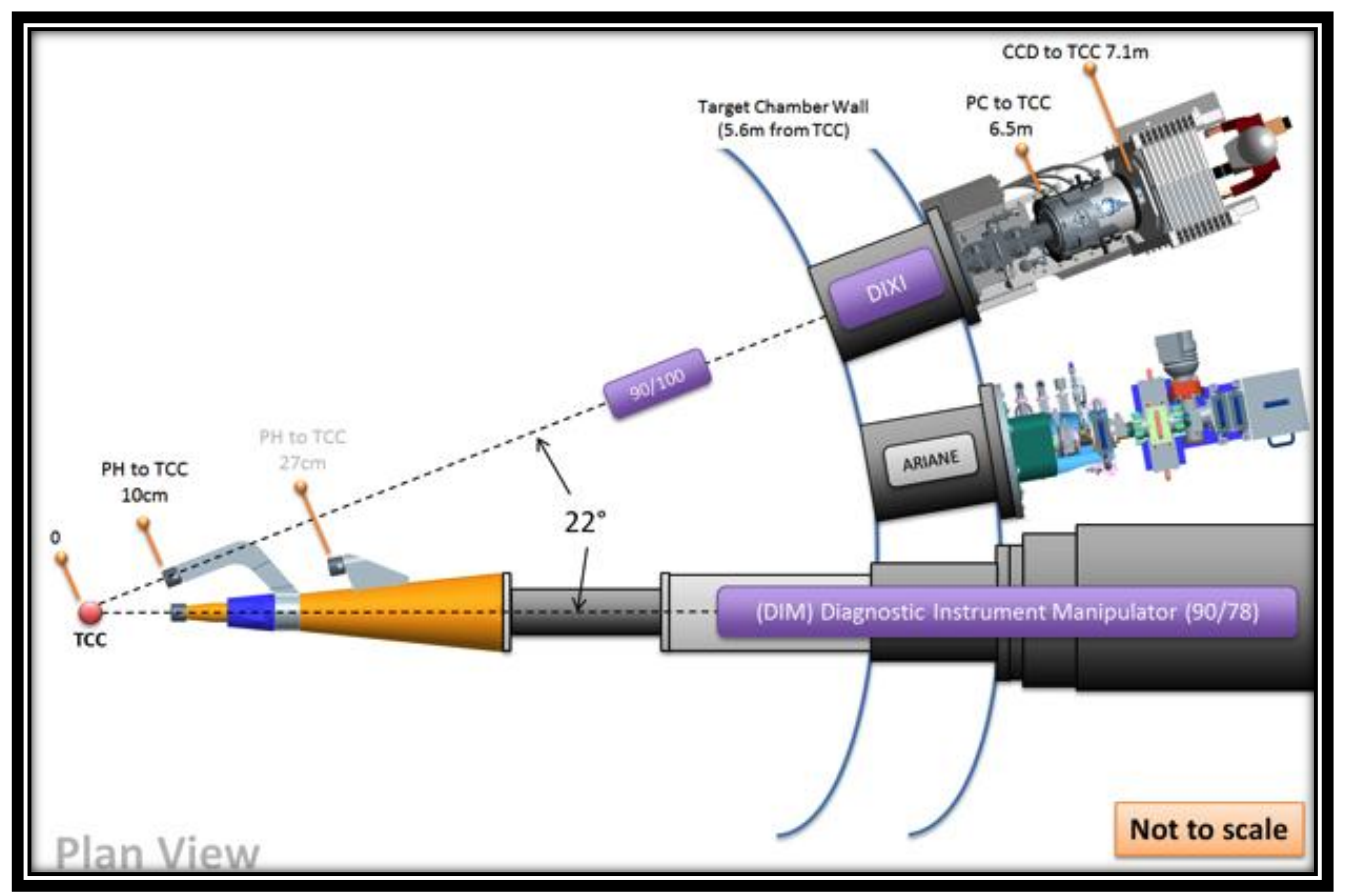

Figure 3. Plan view of imaging configuration

\section{DIXI Cone Design}

The new DIXI cone is based on previous ARIANE designs and includes the following changes. First, the design of the original ARIANE cone included a raised boss on the exterior of the cone for attachment of the added pinhole mount. By optimizing the cone design we were able to reduce the inner diameter of the cone and provide adequate wall thickness for a recessed mounting. This significantly reduces cost and complexity of machining, see figure 4. The reduced pinhole overfill described in section 3 also allows us to reduce the overall pinhole cap size therefore reducing debris load and the cost of tantalum pinhole plates.

The most significant change to the original cone design is the addition of the DIXI pinhole mount. The DIXI mount or arm is able to withstand a $2.0 \mathrm{MJ}$ impulse load and provides nominal placement of the pinholes to better than \pm 250 um of the DIXI detector axis. The design of the pinhole mount and its location of the cone is derived from port survey data resolved using a laser tracker system. The laser tracker system determines the as-built DIXI port coordinates and provides a port vector. With this data we are able to accurately place the pinhole mount in the model with respect to the as-built detector axis when mounted. 


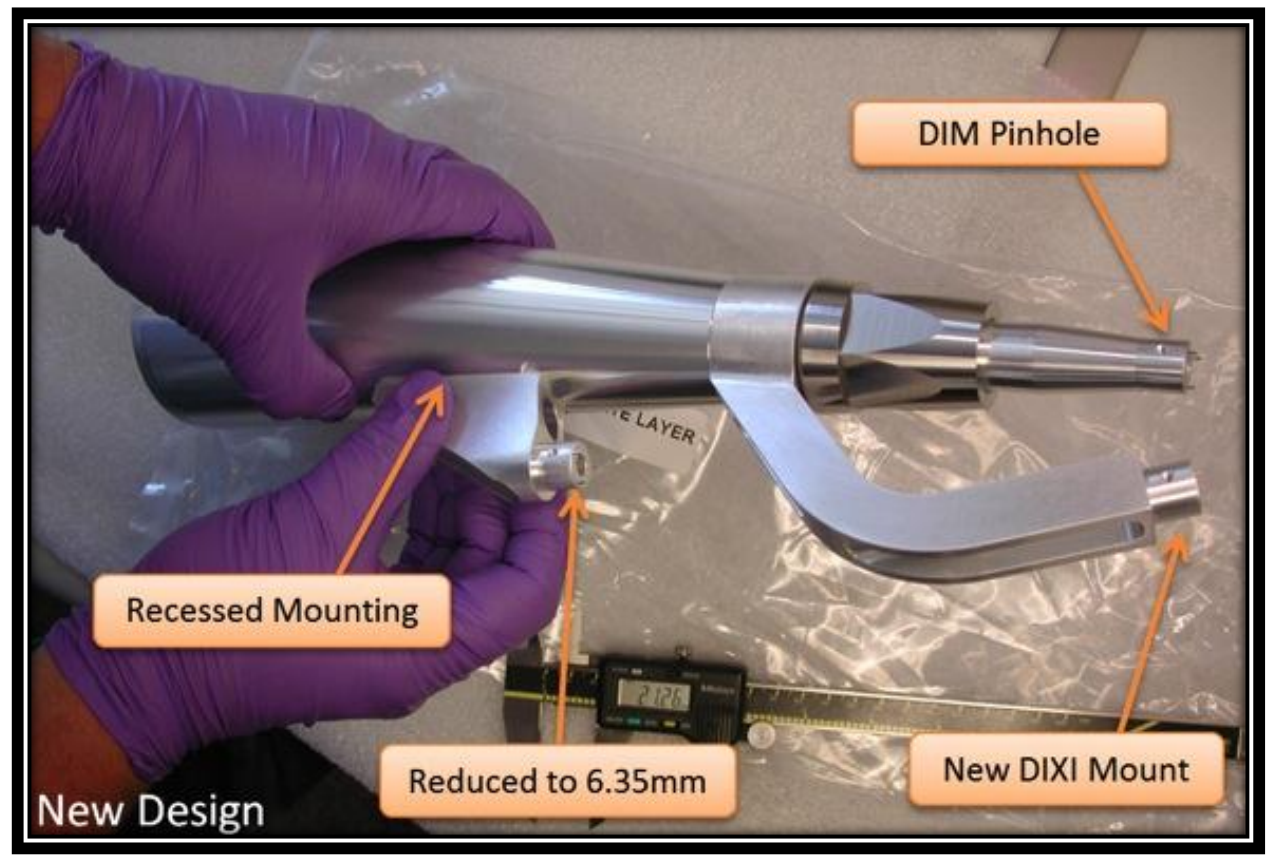

Figure 4. New DIXI cone design with 6.35 ARIANE pinhole mount

\section{DIXI Pinhole Design}

The DIXI instrument is unique in that the gated detector sits $20^{\circ}$ off axis. By positioning the x-ray sensitive backend off axis a typical pinhole configuration which contains collimators to block background signal is not needed. A standard pinhole collimator stack up usually consists of a 75um Ta pinhole plate between a set of 500um Ta collimators, typically with sheets of $125 \mathrm{um}$ thick Kapton to capture spalling off the plates to prevent pinhole closure.

The colliamtors themselves serve three purposes, (1) shield the instrument from background, (2) create a known edge to help de-convolve the gated images and (3) act as a structural support to prevent the thin pinhole plate from being launched and becoming a debris source. In the case of DIXI not only are they not needed but they would also not work. Given DIXI's large 64x magnification an existing three part stack design of collimator-pinhole-collimator would result in as few as 2 to 3 images on a PC strip. The limiting factor to achieve more images with this configuration is the manufacturability of the collimators. As the typical 150um collimator holes get closer the landing between holes becomes too small to handle the heat load generated during laser drilling, melting the landing between holes.

For DIXI a new pinhole platform was conceived using polycarbonate as a backing structure. Polycarbonate was an ideal candidate as it has very low attenuation and is extremely tough. Without collimators the pinhole spacing can now become much denser, even to the point of overlapping the images if not carefully spaced. The new configuration stack consists of 125 um kapton, stainless spacer, $75 \mathrm{um}$ Ta pinhole plate, stainless spacer and $1.3 \mathrm{~mm}$ of polycarbonate. For clarification the spacers allow deformation of the pinhole plate to reduce spall and loading onto the polycarbonate disc. 


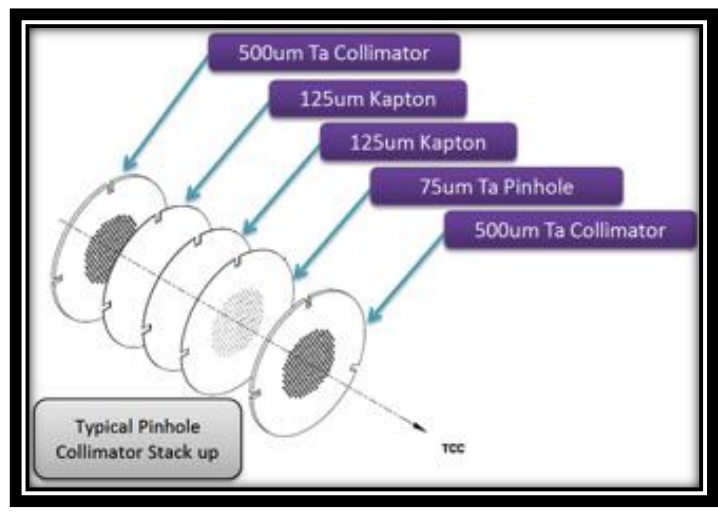

Figure 5. Typical pinhole and collimator stack up

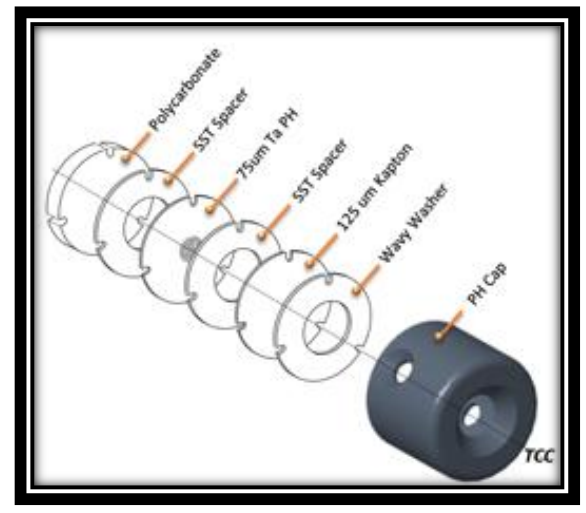

Figure 6. New DIXI configuration

The proposed DIXI pinhole platform has been reviewed by the debris and shrapnel group and was fielded for evaluation in April of 2012 with all 192 beams delivering $1.63 \mathrm{MJ}$ to the target. The test was successful with only minor discoloration to the polycarbonate plate. The stainless spacer showed some signs of damage and the pinhole remained intact with significant debris hits and melting. Post shot images of the test parts are shown in figure 6.

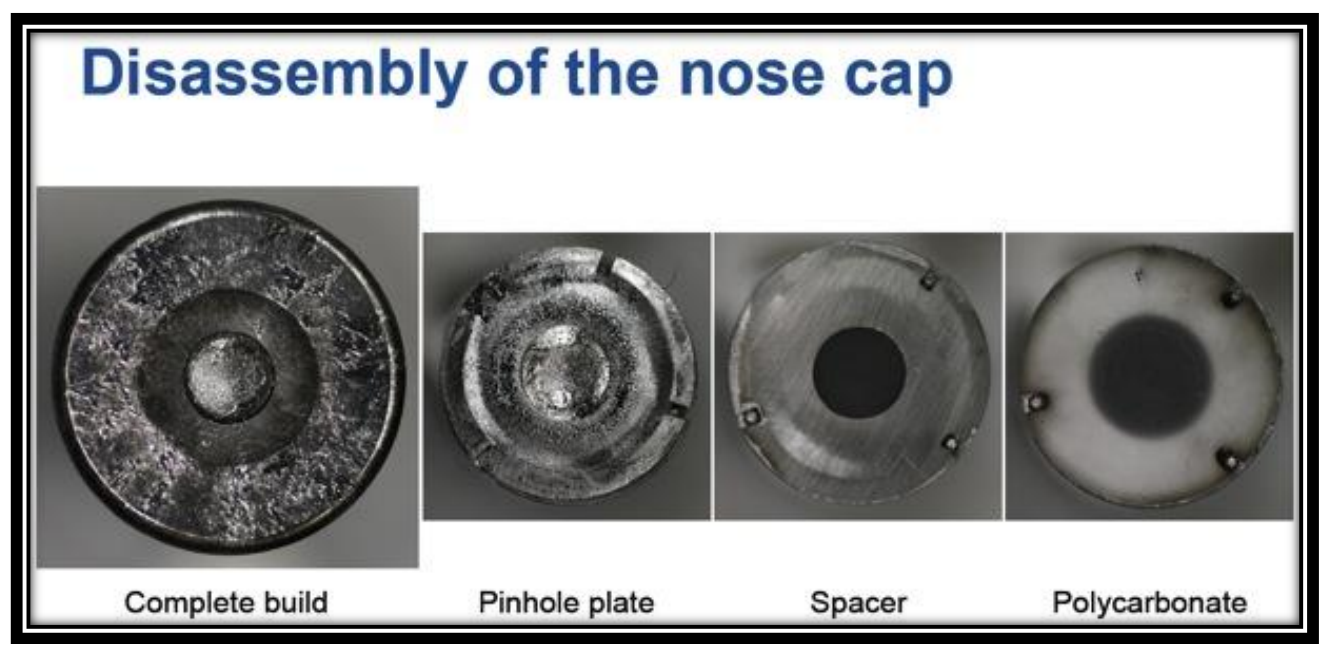

Figure 6. Post shot images of the DIXI pinhole debris test

\section{Filter Image Plate Vessel (FIP)}

The DIXI FIP is based on a previous design used on ARIANE. The FIP vessel sits between the main target chamber gate valve and the DIXI isolation valve. The purpose of the FIP is to allow easy access for adding and removing of filter materials and image plates used to record shot data. The FIP vessel has 2 compartments that are accessible. The first compartment is fitted with an aluminum frame that can hold a variety of filtering and image plate configurations. The front compartment is designed to be accessible in between shots, which allows for set-ups to be exchanged as needed. During removal the 
filter packs are inspected for debris damage. If damage is noted the EMI filter in the next compartment will be removed and inspected.

The EMI shield was designed to prevent unwanted noise induced onto the photocathode. The EMI pulse generated from the TC can alter the high speed photocathode pulse form and distort the signal or image. The EMI shield is a $25 \mathrm{um}$ black kapton base material vapor deposited with $500 \mathrm{~nm}$ of aluminum. The shield is bonded with a conductive adhesive and clamped to a frame. The EMI cassette is a 2 part tapered frame that expands to create an EMI tight seal within the vessel. The EMI design has been fielded and tested on Comet at the Jupiter laser Facility (JLF). See figures $7 \& 8$ for a detailed view of the assemblies.
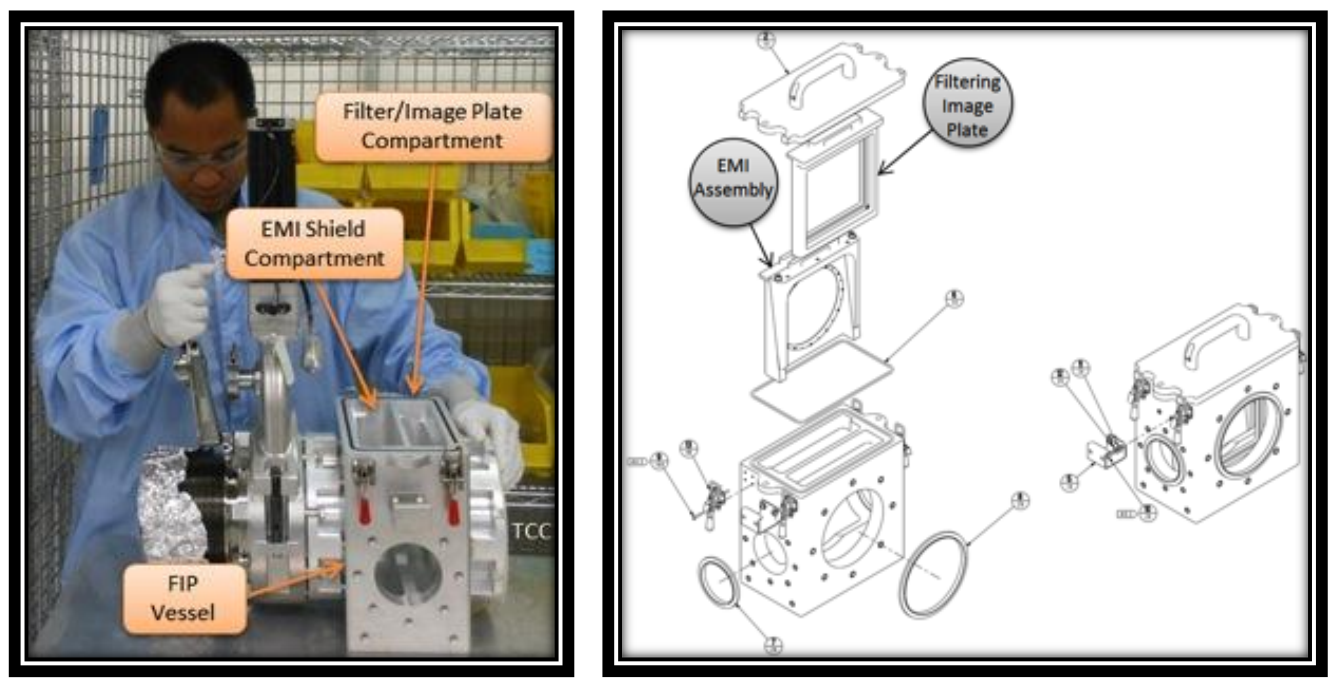

Figure 7. DIXI FIP vessel during initial assembly

\section{Large Format CsI Photocathode}

DIXI uses a large format Cesium Iodide (CsI) photocathode (PC) on the front end. During testing it was evident that improvements were needed in two areas, improved tautness and electrical contact at the strip interface. The flatness of the DIXI photocathode is critical and can only be controlled by ensuring the kapton membrane is taught. Any wrinkling or wave in the plane of the photocathode results in distorted images, Figure 9 and [2]. Additionally the strip lines on the photocathode are aluminum and it was difficult to make good electrical contact at all four strip lines simultaneously. Without sufficient force to break through the oxide layer the electrical contact would fade in and out and was sensitive to the tightening sequence of fasteners around the clamping ring. The clamping ring configuration also induced a radial force inward toward the center of the photocathode which only added to the undesired wrinkling. 


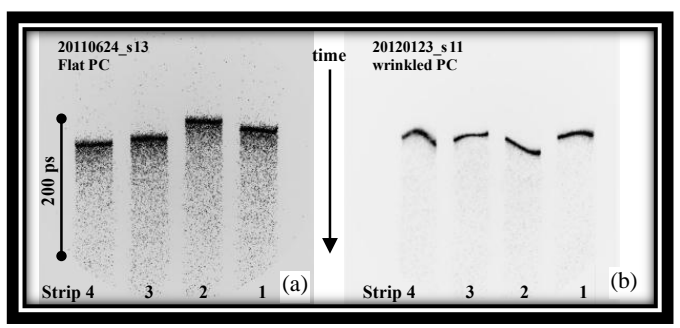

Figure. 9 Sample data showing the difference between a flat photocathode (a) and a wrinkled photocathode (b)

The primary cause of the photocathode wrinkling was the stiffness of the mechanical support ring. The original polyether ether ketone (PEEK) support ring was relatively small in cross section and easily deformed with an applied load, Figure 10. To improve on this the PEEK was exchanged for a Macor ceramic that is $16 \mathrm{x}$ more stiff and the pre-tensing/bonding of the Kapton film was changed, Figure 11.

During electrical breakdown studies at general Atomics (GA) a glass view port was added to capture voltage breakdown with a high speed camera. Instead what was found is the tightening of an initially loose PC after being pumped down for $>12$ hours. It emerged that Kapton is hydrophilic and can absorb as much as $10 \%$ per unit volume of water vapor thus making it grow in size. For the final design we took advantage of both thermal effects and humidity during bonding of the Kapton to the Macor ring. The Kapton film is first bonded to an oversized Delrin ring. The Delrin and Kapton assembly is then placed over the Macor frame in an environmental oven and bonded at $50^{\circ} \mathrm{C}$ and $60 \%$ humidity. The Delrin ring expands due to elevated temperatures and the Kapton grows from the high humidity. It remains in this state until the Kapton film is fully cured to the Macor frame. Once removed from the environmental oven the Delrin set up frame is trimmed away and the film tautness only increases more as water vapor is driven off. Once installed and pumped down in DIXI the remaining water vapor is driven off, further increasing the film tautness.

To improve on the electrical contacts the aluminum strip lines are argon etched to remove the oxide layer and a thin layer of gold is vapor deposited for increased reliability.

The end result is a flat tight photocathode that can be removed and installed with stable electrical connections. The new configuration was installed and has been running reliably for a period of 1.5 years at which time the PC was exchanged with a new PC of the same design due to efficiency degradation of the Cesium Iodide.

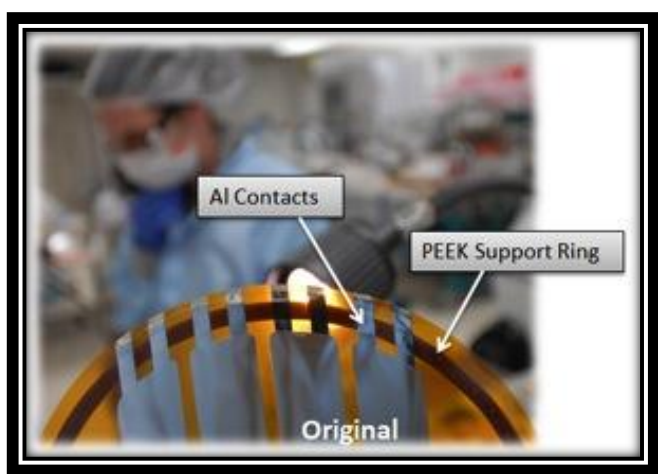

Figure 10. Original photocathode configuration with aluminum strip contacts and PEEK support frame

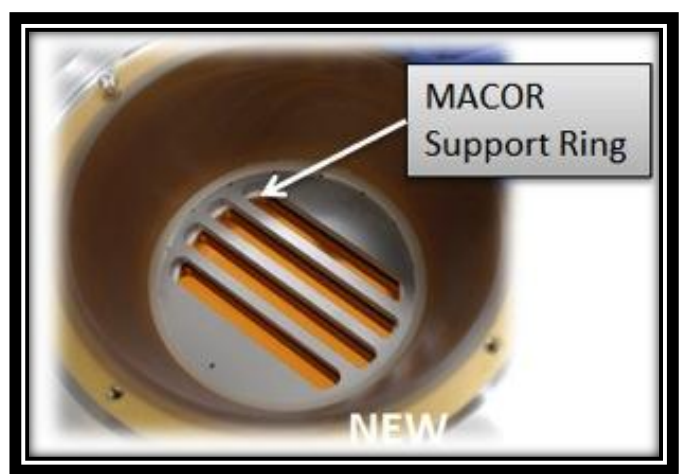

Figure 11. New photocathode shown installed in the DIXI with gold contacts, new Macor support frame and revised tensioning/bonding procedure 


\section{DIXI Instrument And Detector}

DIXI looks at X-rays that are driven off the target as it implodes. A pinhole array projects multiple images of the incoming X-ray signal onto the plane of the PC. As the X-rays pass through the PC, DIXI is timed to send a high voltage ramp along the PC strip during peak compression of the target. As the ramp propagates along the strip electrons are liberated off the CsI PC. The electrons liberated off the PC have an energy distribution dependent on the pulse form.

While the electrons travel along the drift space the 4 electromagnetic coils generate a longitudinal Bfield that guides the electrons and the signal stretches as the higher energy electrons are traveling at a faster velocity. By stretching or dilating the signal the effective gating at the MCP is reduced by a factor of 7 over conventional gated imagers and provides $<10$ ps temporal resolution, Figure 12, [1-4].

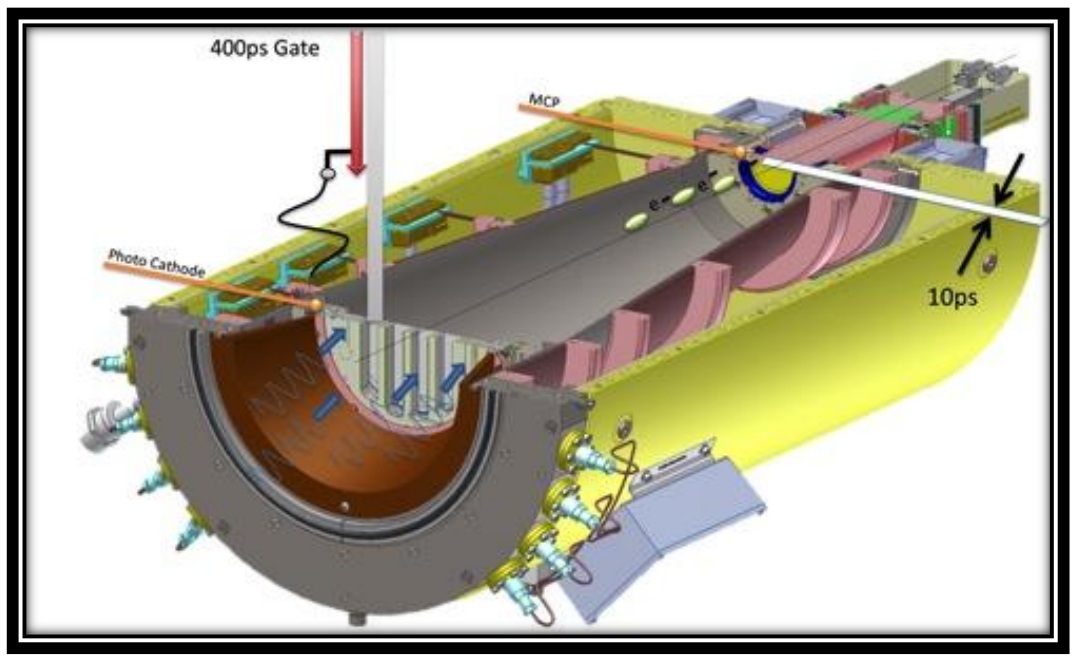

Figure 12. Section view of DIXI with illustrations that show the dilated signal as it traverses along the drift tube. Effective gating at MCP is reduced by a factor of 7 over typical gated imagers.

\section{EMI Testing}

Prior to deployment on NIF DIXI has undergone extensive testing and calibration at the Jupiter Laser Facility (JLF). As part of this testing DIXI had to be surveyed to determine whether it met the allowable EMI levels for operation in the target bay. DIXI had several areas that needed to be checked for potential EMI sources, PC cables, PC, electromagnetic cables, electromagnet coils, micro channel plate (MCP) strip lines (carries the MCP pulse) and the MCP itself, Figure 13. 


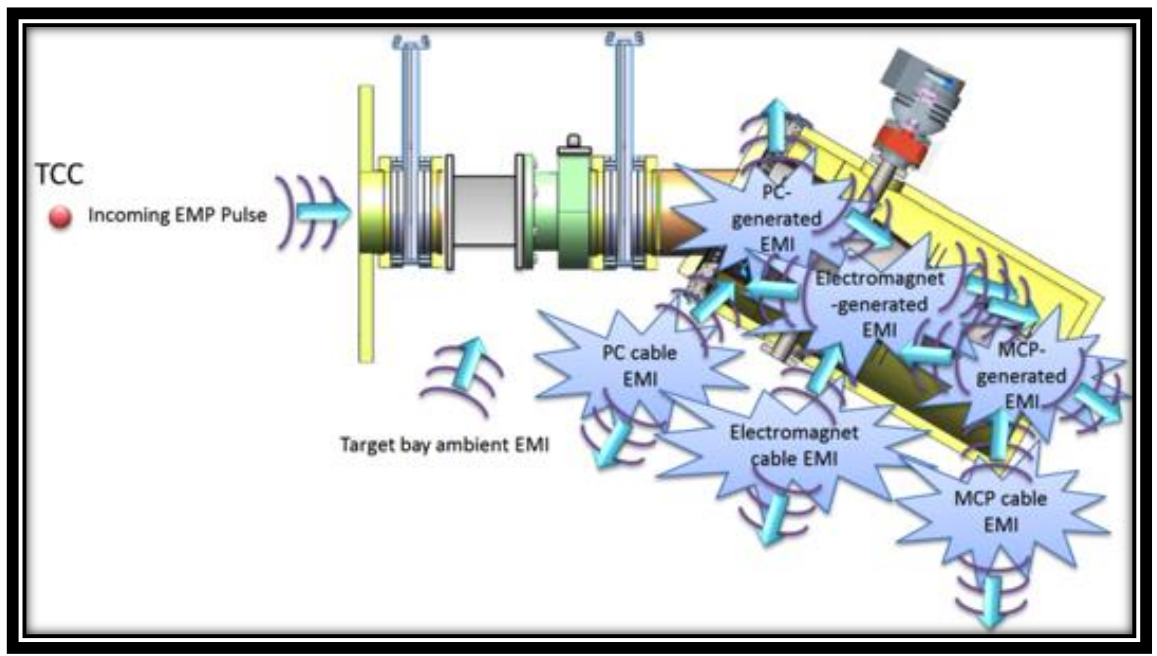

Figure 13. Potential EMI and EMP sources on DIXI

The largest EMI source measured was near the MCP strip lines. The original configuration offered no EMI protection and measurements as high as 30 gauss were recorded adjacent to the strip line safety cover. To mitigate this, the entire backend electronics with separate drive boxes was redesigned to encompass the MCP strip lines and electronics in an EMI tight enclosure, Figure 14 \& 15. Other changes were the addition of a CCD EMI enclosure, upgraded shielded cables, conduits, and bulkheads connections(?) where needed. Testing of the magnetic field revealed that the stray field levels were within the acceptable values and no additional shielding was needed.

The most sensitive instrument near DIXI in the target bay is the photo multiplier tubes (PMT) on the Filter Fluorescer Hard X-Ray Spectrometer (FFLEX). To certify DIXI would not impact the signals on FFLEX a PMT tube was positioned near DIXI in the same orientation and distance as in the target bay. Background measurements on the PMT from DIXI EMI were recorded at the JLF facility. To simulate the worst case operating conditions all potential sources were pulsed. The resulting EMI upgrades yielded a 10x reduction of the noise picked up by PMT test setup, Figure 16.
Comment [SN1]: I believe that was from the stray magnetic field measurement, rather than the measurement from the mcp noise...

Also, this is not consisten with the measurements in figure 16!

Formatted: No underline 


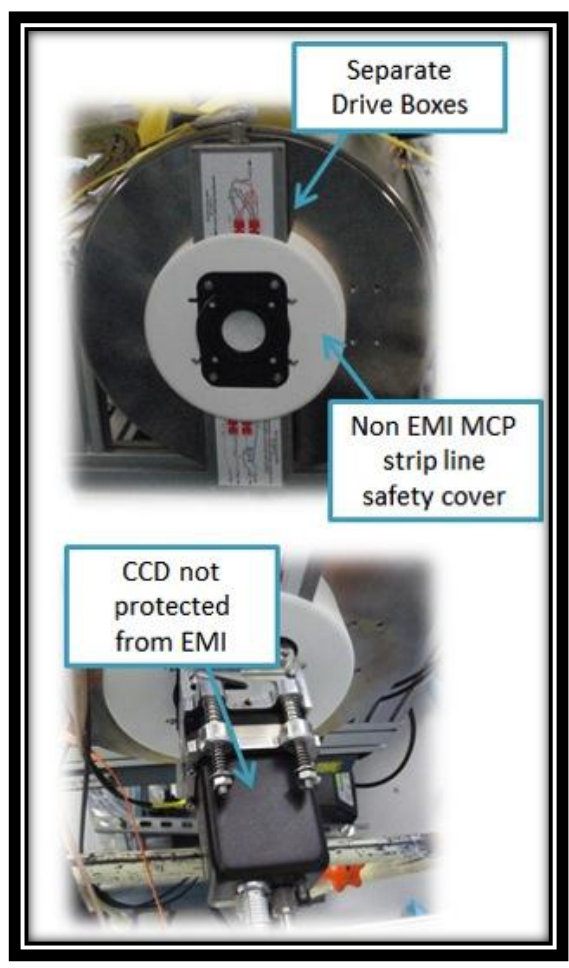

Figure 14. Original backend configuration with separate drive boxes

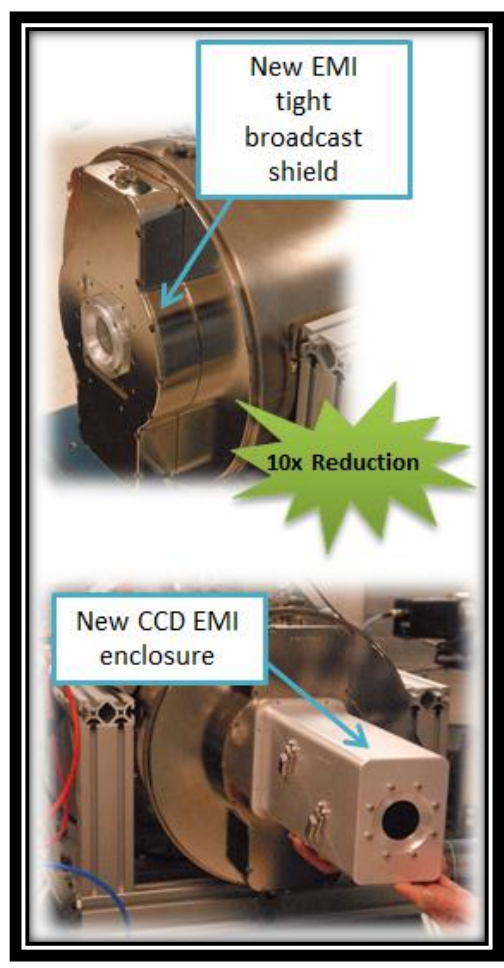

Figure 15. Updated Configuration with new integrated EMI broadcast shield and electronics

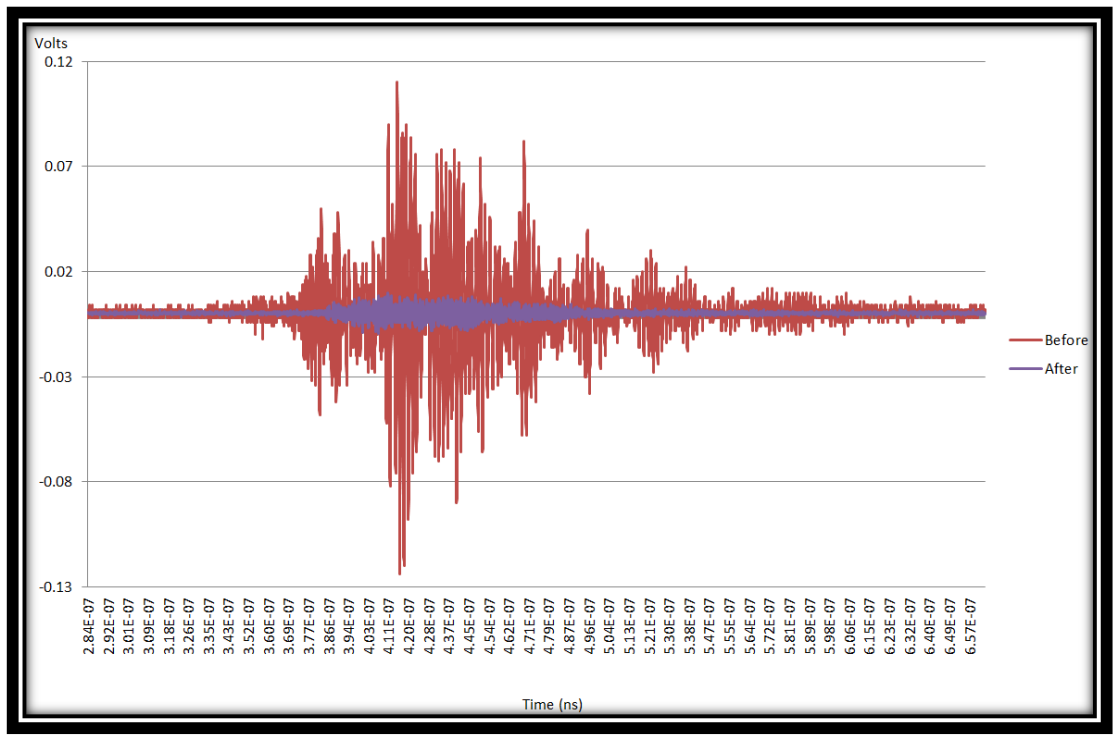

Figure 16.

PMT

background

recordings

that show the

$10 x$ reduction in EMI noise

after

installation of the updated design. 


\section{DIXI Neutron Shielding}

To keep up with the increasing target yield new diagnostics with improved rad hardness are needed. DIXI is capable of collecting data on CCD at $\mathrm{Yn} \sim 10^{16}$ with the use of extensive shielding. As previously described in section 2 the DIXI detector sits below the direct line of site of the port and is shielded by the 18" of target chamber Gunite. Directly outside the target chamber wall in line of site of the detector there is an added $30 \mathrm{~cm}$ of polyethylene direct shine shielding. Additional filtering consisting of $5 \mathrm{~cm}$ of lead and $20 \mathrm{~cm}$ of polyethylene surrounds the detector at the backend on 5 sides.

The lead and polyethylene shielding directly around the detector is configured into individual drawers. There are a total of 18 drawers that can be easily opened to allow access to the detector for maintenance or removal/exchange of film, Figures $17 \& 18$. The shield drawers are supported by a heavy duty linear slide and guided by a set of cam followers on the top rail. The shield drawer slides are fitted with integrated locks that automatically lock the drawers in the closed and open position for safety.
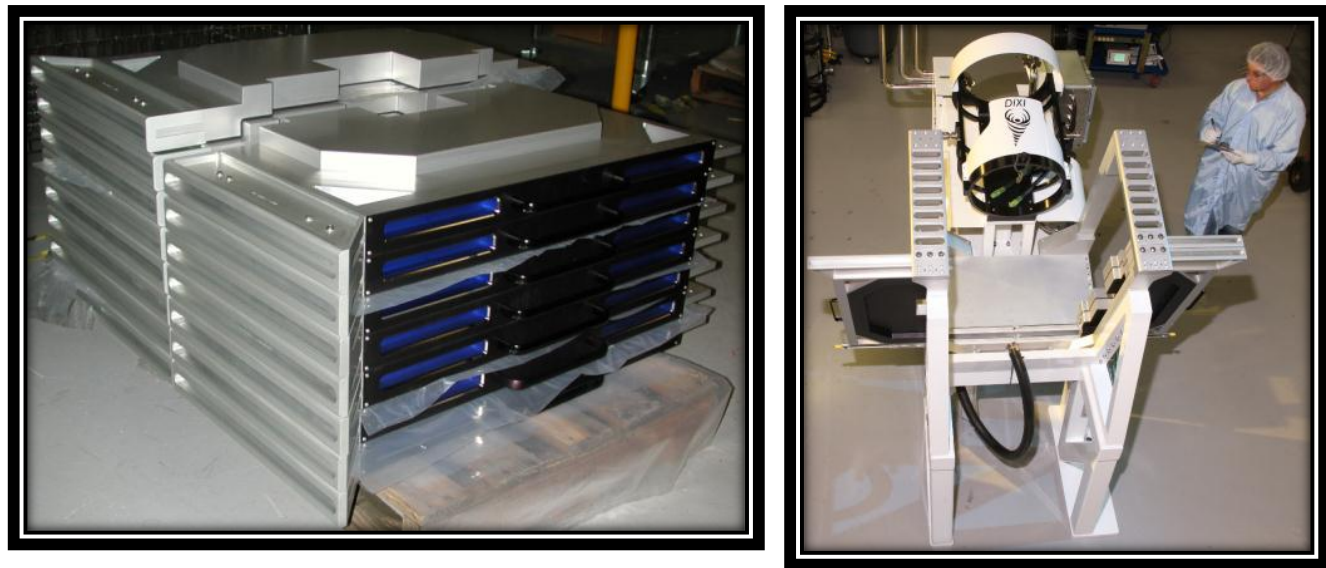

Figure 17. DIXI shield drawers fully assembled waiting installation

Figure 18. DIXI with $3 \mathrm{x}$ shield drawers installed for testing. Shown in the open position.

The DIXI shield drawers are constructed using MIC6 cast aluminum tooling plate. Cast plate was used for the ease of machining and dimensional stability needed to maintain tight gaps between drawers. There are 3 shielding components in each drawer, the $5 \mathrm{~cm}$ lead insert and (2) $20 \mathrm{~cm}$ thick poly inserts. The poly insert is manufactured in two pieces to utilize standard stocked material sizes. The top side of the shield drawer is fitted with an aluminum cover to keep the poly $90 \%$ leak tight. The aluminum shell and sheet metal covers are required to meet combustible fuel requirements for the NIF fire suppression system. The shield drawers are nested to block direct line of sight through the gap between drawers, Figure 19. Since the drawers are nested they have to be opened and closed in sequence, starting from one end. This drawer setup of the DIXI shielding configuration allows easy access without the need for heavy rigging equipment. 


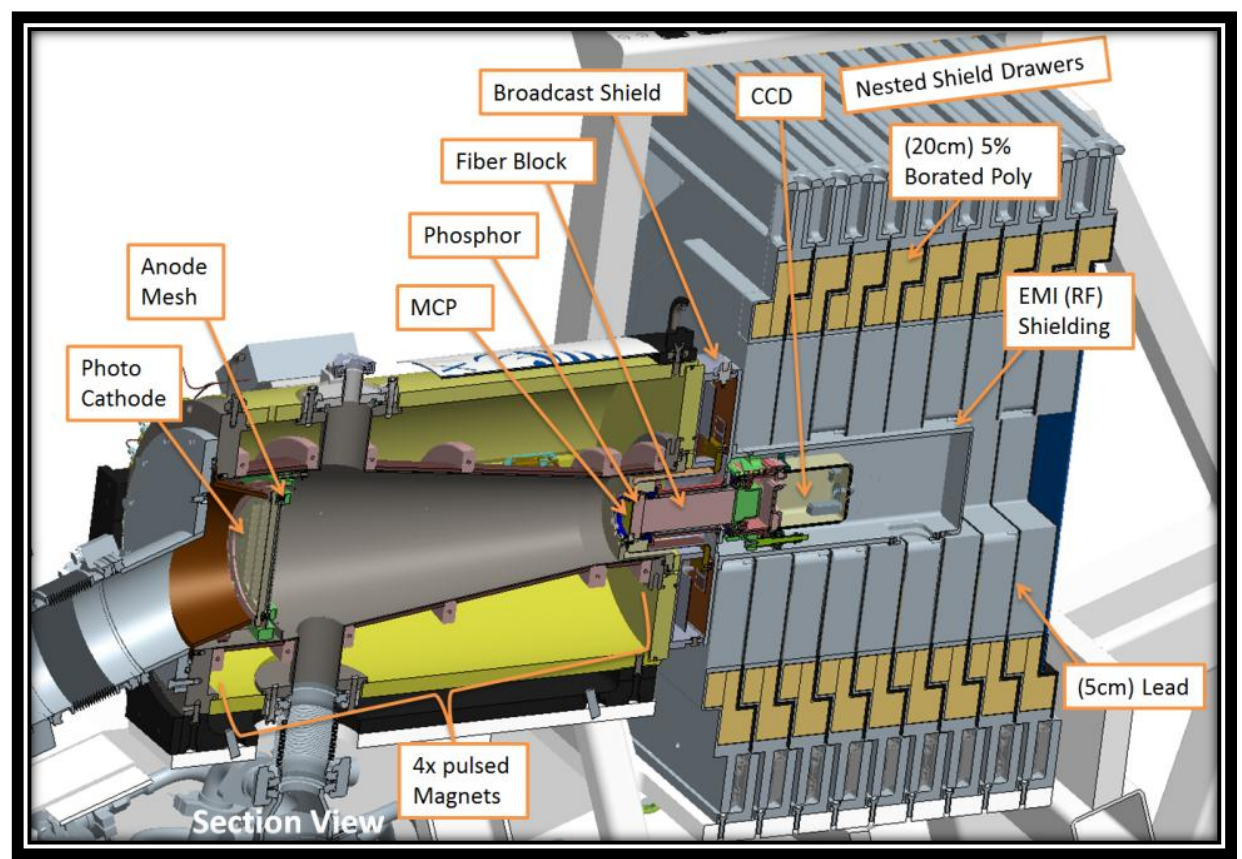

Figure 19. Cross section of DIXI shields drawers showing the nested geometry.

\section{DIXI Status}

The design of the DIXI infrastructure is completed and the system hardware assembly is $90 \%$ complete. The lower left image, figure 20 shows a side view of the system during assembly, all of the aesthetic panels are in place therefore the vacuum hardware is not shown. The DIXI mounting harness is visible, note the DIXI diagnostic is not currently installed in this image. The EMI conduits for the high voltage cables for the electromagnets are visible as well. The magnet and vacuum junction boxes are shown attached to the front utilities stand. The tritium UDP panel for tritium sampling is also shown on the rear shielding stand. There are 3 of the 18 shield drawers installed in the open position. For safety reasons not all $1500 \mathrm{lbs}$ of shielding can be installed until the frame is anchored down. 


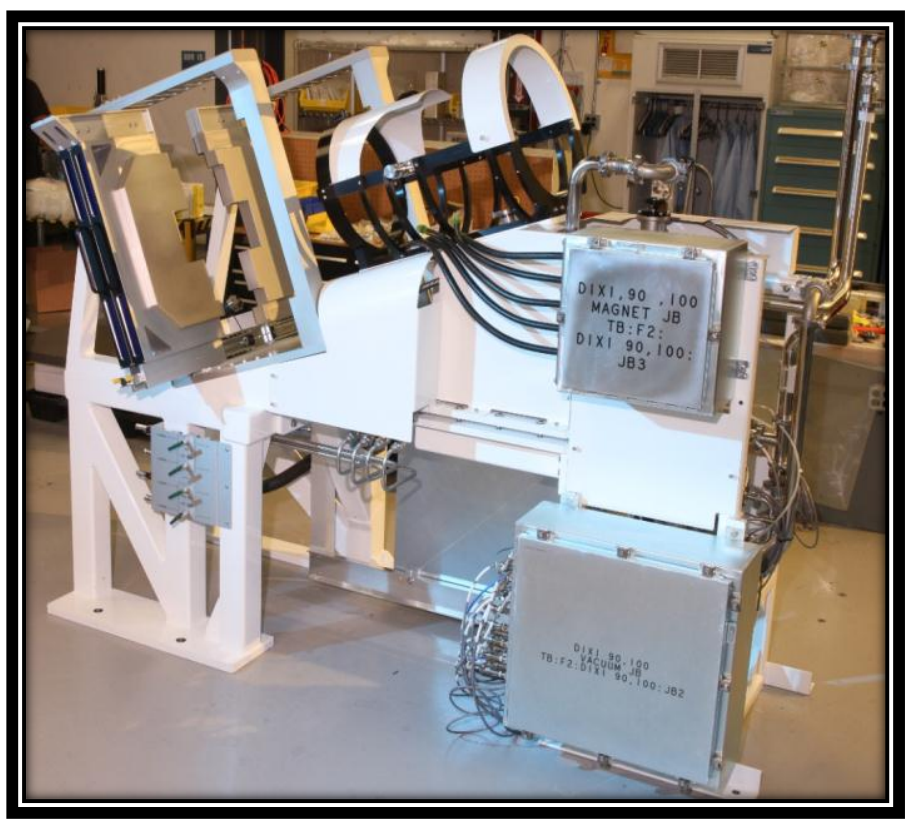

Figure 20. The DIXI support and infrastructure during assembly for use in NIF

The DIXI infrastructure in figure 20 has been surveyed into location and installed in the NIF. Final hardware and utilities installation in the NIF facility is $50 \%$ complete. All utilities, electrical and NIF facility modification are complete. DIXI testing and calibration at the Jupiter Laser Facility is complete. DIXI is expected to be ready for shots on the NIF January 2014.

Lawrence Livermore National Laboratory is operated by Lawrence Livermore National Security, LLC, for the U.S. Department of Energy, National Nuclear Security Administration under Contract DEAC52-07NA27344. Work supported by U.S. Department of Energy under Contract DE-AC52-06NA27279.

[1] T. J. Hilsabeck et al., Rev. Sci. Instrum., 81, 10E317, (2010)

[2] Nagel, S .R., Ayers, M. J., Felker, B., Hilsabeck, T. J., Chung, T., Smith, R. F., Bell, P. M., Bradley, D. K., Collins, G. W., Kilkenny, J. D., Sammuli, B., Hares, J. D. and Dymoke- Bradshaw, A. K. L.,"Perforemance measurements of DIXI (dilation x-ray imager) photocathode using a laser produced $\mathrm{x}$-ray source," Proc of SPIE, 8505, 85050H-2 (2012).

[3] Nagel, S .R., Hilsabeck, T. J., Bell, P. M., Bradley, D. K., Ayers, M. J., Barrios, M. A., Felker, B., Smith, R. F., Collins, G. W., Jones, O. S., Kilkenny, J. D., Chung, T., Piston, K., Raman, K. S., Sammuli, B., Hares, J. D. and Dymoke-Bradshaw, A. K. L., "Dilation x-ray imager a new/faster gated x-ray imager for the NIF," Rev. of Sci. Instrum., 83, 10E116 (2012); http://dx.doi.org/10.1063/1.4732849.

[4] Nagel, S .R., Ayers, M. J., Felker, B., Hilsabeck, T. J., Chung, T., Bell, P. M., Bradley, D. K., Collins, G. W., Kilkenny, J. D., Hares, J. D. and Dymoke- Bradshaw, A. K. L.,"2D magnetic field warp reversal in images taken with DIXI (dilation x-ray imager)," Proc of SPIE, 8850, 8850-17 (2013).

[5] G.A. Kyrala, S. Dixit, S. Glenzer, D. Kalantar et al., Rev. Sci. Instrum. 81 10E316 (2010).

[6] G. H. Miller et al., Nucl. Fusion 44, S228 (2004).

[7] E. I. Moses et al., Phys. Plasmas 16, 041006 (2009).

[8] Ayers, M. J. et al., "Design and implementation of high magnification framing camera, ARIANE" Proc. Of SPIE Vol. 8505 85050J-1 